\title{
Educación para la mitigación y adaptación al cambio climático en América Latina ${ }^{1}$
}

\section{Norella Cruz Castaño}

https://orcid.org/0000-0003-3215-084X Universidad Pedagógica Nacional,

Colombia

ncruzc@upn.edu.co

\section{Pablo Páramo}

https://orcid.org/0000-0002-4551-3040 Universidad Pedagógica Nacional, Colombia

pparamo@pedagogica.edu.co

\section{Resumen}

Este artículo establece el estado actual de la investigación en educación sobre cambio climático en Latinoamérica, a partir de una revisión de las publicaciones que aparecen en las bases de datos Scielo, Ebsco Host, Dialnet y Redalyc, desde 2009 a 2019. Se utilizaron las categorías "educación" Y "cambio climático" o "calentamiento de la tierra" y se seleccionó una muestra de 36 estudios. Los resultados indican que en América Latina la educación sobre el cambio climático se ha abordado principalmente con estudios de percepción y representaciones sociales y en algunos casos con propuestas educativas que tienen a la población joven como objetivo. Se evidencian concepciones erróneas sobre el cambio climático y confusión de este fenómeno con otras problemáticas ambientales.

\section{Palabras clave (Fuente: tesauro de la Unesco)}

América Latina; cambio climático; currículum escolar; educación ambiental; politicas ambientales.

$1 \quad$ Este artículo es derivado de la tesis de doctorado "Valoraciones sobre el cambio climático en estudiantes universitarios colombianos: atribución de responsabilidades, relevancia frente a otras problemáticas mundiales y acciones individuales", para sustentar en la Universidad Pedagógica Nacional, Bogotá, Colombia, en el Doctorado Interinstitucional en Educación (DIE).

Recepción: 04/09/2019 | Envío a pares: 22/1/2020 | Aceptación por pares: 15/07/2020 | Aprobación: 26/08/2020 


\title{
Education for Climate Change Mitigation and Adaptation in Latin America
}

\begin{abstract}
This article establishes the current state of research into climate change education in Latin America based on a review of publications in the Scielo, Ebscohost, Dialnet, and Redalyc databases from 2009 to 2019. The categories "education" AND "climate change" OR "global warming" were used, and a sample of 36 studies was selected. The results show that in Latin America, climate change education has been approached mainly by studies of perception and social representations, including educational proposals that target the young population. Misconceptions about climate change and confusion of this phenomenon with other environmental problems are noted.
\end{abstract}

Keywords (Source: Unesco Thesaurus)

Latin America; climate change; school curriculum; environmental education; environmental policies. 


\section{Educação para a mitigação e adaptação à mudança climática na América Latina}

Resumo

Este artigo estabelece o estado atual da pesquisa em educação sobre mudança climática na América Latina, a partir de uma revisão das publicações que aparecem nos bancos de dados Scielo, Ebscohost, Dialnet e Redalyc, de 2009 a 2019. Utilizaram-se as categorias "educação" $E$ "mudança climática" OU "aquecimento da terra", e selecionou-se uma amostra de 36 estudos. Os resultados mostram que na América Latina a educação sobre a mudança climática abordou-se principalmente com estudos de percepção e representações sociais; alguns com propostas educacionais que têm a população jovem como objetivo. Evidenciam-se concepções errôneas sobre a mudança climática e a confusão desse fenômeno com outras problemáticas ambientais.

\section{Palavras-chave (Fonte: tesauro da Unesco)}

América Latina; mudança climática; currículo escolar; educação ambiental; politicas ambientais. 
El cambio climático (CC), después de la pandemia del Covid-19, es el tema de mayor preocupación en la comunidad internacional, quizás más que las guerras en algunos países, por incidir a nivel mundial sobre aspectos económicos y sociales (Duarte, 2014). Actualmente hay consenso científico sobre su existencia (Boussalis y Coan, 2016) y el Intergovernmental Panel on Climate Change (IPCC, 2007; 2014; 2018) ha confirmado el papel de la acción humana en la generación del fenómeno.

A pesar de que su causa es antropogénica, el CC no ha trascendido a acciones sociales (Meira et al., 2009). Las encuestas han puesto de manifiesto que un alto porcentaje de ciudadanos de varios países no tienen el CC entre sus principales preocupaciones, $\mathrm{ni}$ siquiera creen que los científicos se hayan puesto de acuerdo sobre su existencia y dudan del papel que pueden adoptar las personas para mitigar sus efectos (Boussalis y Coan, 2016; Clayton et al., 2015).

Para hacer frente al CC hace falta más que la ciencia, porque se requiere el apoyo de la sociedad y de políticas públicas. América Latina y el Caribe aportan entre el $9 \%$ y el 10\% de las emisiones totales de gases de efecto de invernadero (GEI), pero no son emisores históricamente importantes. Sin embargo, sí es una región vulnerable a sus efectos (Cepal, 2014). Especialmente el impacto es mayor en poblaciones de ingresos bajos debido a que dependen de una sola fuente de ingreso, tienen menor educación, son familias numerosas y poseen escasas fuentes de financiación (Cecchini et al., 2012). La adaptación y la mitigación son estrategias complementarias para reducir y gestionar los riesgos del cambio climático (IPCC, 2014). En América Latina, las estrategias de mitigación han estado encaminadas a la implementación de mecanismos de desarrollo limpio, al sector energético, al transporte y a los sectores agropecuario, forestal y de residuos, y las de adaptación están relacionadas con el sector forestal, la biodiversidad, el sector agropecuario, el recurso hídrico, la infraestructura, los asentamientos humanos, la salud, los recursos energéticos y la ocurrencia de eventos extremos
(Cepal y Unión Europea, 2017). Es de resaltar que no se menciona la educación en las políticas de mitigación y adaptación para América Latina. No obstante, como política educativa internacional se ha venido trabajando en la acción para el empoderamiento climático, artículo 6 de la Convención Marco de las Naciones Unidas para el Cambio Climático (cMNucc) realizada en 1992, ratificada por varios países en el protocolo de Kioto de 1997 y el Acuerdo de París de 2016.

El seguimiento a esta política educativa ha sido liderado en su mayoría por la Organización de las Naciones Unidas y sus organismos, como la Organización de las Naciones Unidas para la Educación, la Ciencia y la Cultura (Unesco); el Programa de las Naciones Unidas para el Medio Ambiente (Pnuma); el Instituto de las Naciones Unidas para la Formación Profesional y la Investigación (Unitar); el Fondo de las Naciones Unidas para la Infancia (Unicef); la Organización de las Naciones Unidas para la Alimentación y la Agricultura (FAO); y el Fondo Internacional de Desarrollo Agrícola (FIDA), junto con organizaciones no gubernamentales ONG y la Alianza de las Naciones Unidas para la Educación en Cambio Climático. Estas entidades dirigen esfuerzos para poner en marcha soluciones de adaptación y mitigación a través de la educación, la formación y la conciencia pública de los países frente a las problemáticas del cambio climático (Unesco y unfccc, 2016; Unesco, 2017). Sin embargo, la educación sigue estando infrautilizada como recurso estratégico para mitigar el cambio climático y adaptarse a él. Las partes interesadas en la educación de muchos países todavía no han elaborado un marco coherente para la educación sobre el cambio climático (Mochizuki y Bryan, 2015). Colombia ha adoptado las políticas internacionales para el CC y, entre ellas, las educativas. En consecuencia, realizó la estrategia de educación, formación y sensibilización de públicos sobre cambio climático en 2010, la cual fue evaluada en 2017 para hacer seguimiento a su implementación en el documento "Acciones de educación, formación y sensibilización de públicos en cambio climático en 
Colombia". Llama la atención que allí se señala el bajo presupuesto para la educación y sensibilización en el tema de CC, así como la falta de recurso humano para cubrir todos los sectores y públicos a nivel nacional, a lo que habría que agregar que el lenguaje técnico en el que se presenta la información dificulta su consulta y aplicación para la toma de decisiones y que carece de herramientas divulgativas y educativas diferenciadas por públicos y de continuidad en las políticas institucionales (Ideam et al., 2017). Las herramientas divulgativas han sido subsanadas en parte a través de la página web de Minambiente (https://www.minambiente.gov.co/index.php/cambio-climatico).

El escenario climático actual exige que la sociedad redefina el estilo de vida y se promuevan cambios de actitudes individuales y colectivas en la relación con el medio ambiente. La educación ha sido un instrumento usado para facilitar la adaptación de las personas y las sociedades a los desafíos actuales (Heras, 2015), por lo cual juega un papel importante para el CC (Anderson, 2012; Unesco, 2009; 2010; 2017; Unesco y unfCCc, 2016). Sin embargo, los educadores se enfrentan a muchos desafíos cuando se enseña sobre el CC y no hay un amplio acuerdo sobre qué estrategias son las más efectivas.

Los docentes intentan transmitir información precisa a través de programas escolares; no obstante, abundan los conceptos erróneos sobre las causas del CC (Chen, 2011; Choi et al., 2010; Sterman, 2011) y la mayoría de los jóvenes no entienden la ciencia básica del clima (Leiserowitz et al., 2011; Taber y Taylor, 2009). Cuando los alumnos realizan sus propias investigaciones y cooperan con expertos, se ha evidenciado éxito, sobre todo si se basan en teorías transdisciplinarias y/o constructivistas, y es aún mayor cuando se combinan ambos enfoques (Keller et al., 2019).

La revisión sistemática más actualizada sobre educación en CC la realizaron Monroe et al. (2017) y estuvo orientada a revisar investigaciones educativas formales e informales en países anglosajones, en las que se haya evaluado la efectividad de las inter- venciones con miras a generar cambios de actitudes y comportamiento. Los autores revisaron la base de datos académica Ebsco Host, donde identificaron 49 investigaciones que cumplieron los criterios de centrarse en la evaluación de las intervenciones educativas sobre el CC. Entre las intervenciones educativas se identificaron dos estrategias: 1) hacer que la información sobre el CC sea personalmente relevante y significativa para los estudiantes y 2 ) involucrar a los estudiantes en las actividades. En el estudio mencionan que lograron identificar muy pocos programas educativos que aborden intencionalmente el CC con base en disciplinas sociales y científicas (multidisciplinarias, interdisciplinarias o transdisciplinarias) y llaman la atención sobre la importancia de una revisión de las políticas y programas educativos por países o regiones.

A pesar de sus importantes hallazgos, estos no pueden generalizarse a todas las regiones del mundo, por considerar que en educación ambiental las investigaciones han mostrado que existen diferencias culturales importantes (Anderson, 2012), razón por la cual vale la pena preguntarse qué se está haciendo en educación para la mitigación y adaptación al CC en América Latina. Este artículo se centra en la revisión de la producción científica en educación que aborde el CC en los países de esta región. Llevar a cabo esta revisión permite conocer el estado actual de la educación, ver qué tan preparada pueden estar nuestra sociedad para enfrentar el CC, establecer lo que la investigación arroja acerca de cómo se está pensando el problema y orientar estudios que emprendan acciones educativas que contribuyan a la mitigación y adaptación al CC.

\section{Método}

Se realizó una revisión sistemática de documentos en las bases de datos: Scielo, Ebsco Host (fuente académica), Dialnet y Redalyc, a partir de los términos: "education" AND "climate change" OR "Global Warming", seleccionando los últimos 10 años (2009-2019), en la producción científica de los países 
latinoamericanos presentes en dichas bases. Sobre estos resultados, se realizó la lectura de los títulos y resúmenes y se localizaron aquellos que cumplieran con los criterios de "inclusión" y de "exclusión".

En cuanto a los criterios de inclusión, corresponden a artículos publicados entre 2009 y 2019 que traten de alguna manera el tema de la educación para la mitigación y adaptación al CC por parte de instituciones educativas y de otras entidades en países latinoamericanos. Como criterios de exclu- sión, se descartaron de la exploración inicial los artículos que no incluyeran temáticas relacionadas con educación para la mitigación y adaptación al CC, ni pertenecieran a los países latinoamericanos y que fueran realizados antes de 2009 .

\section{Resultados}

A continuación, se presenta el prisma que indica cómo se realizó la selección de artículos según el método ya descrito para esta revisión (Figura. 1).

Figura 1. Prisma para la selección de artículos

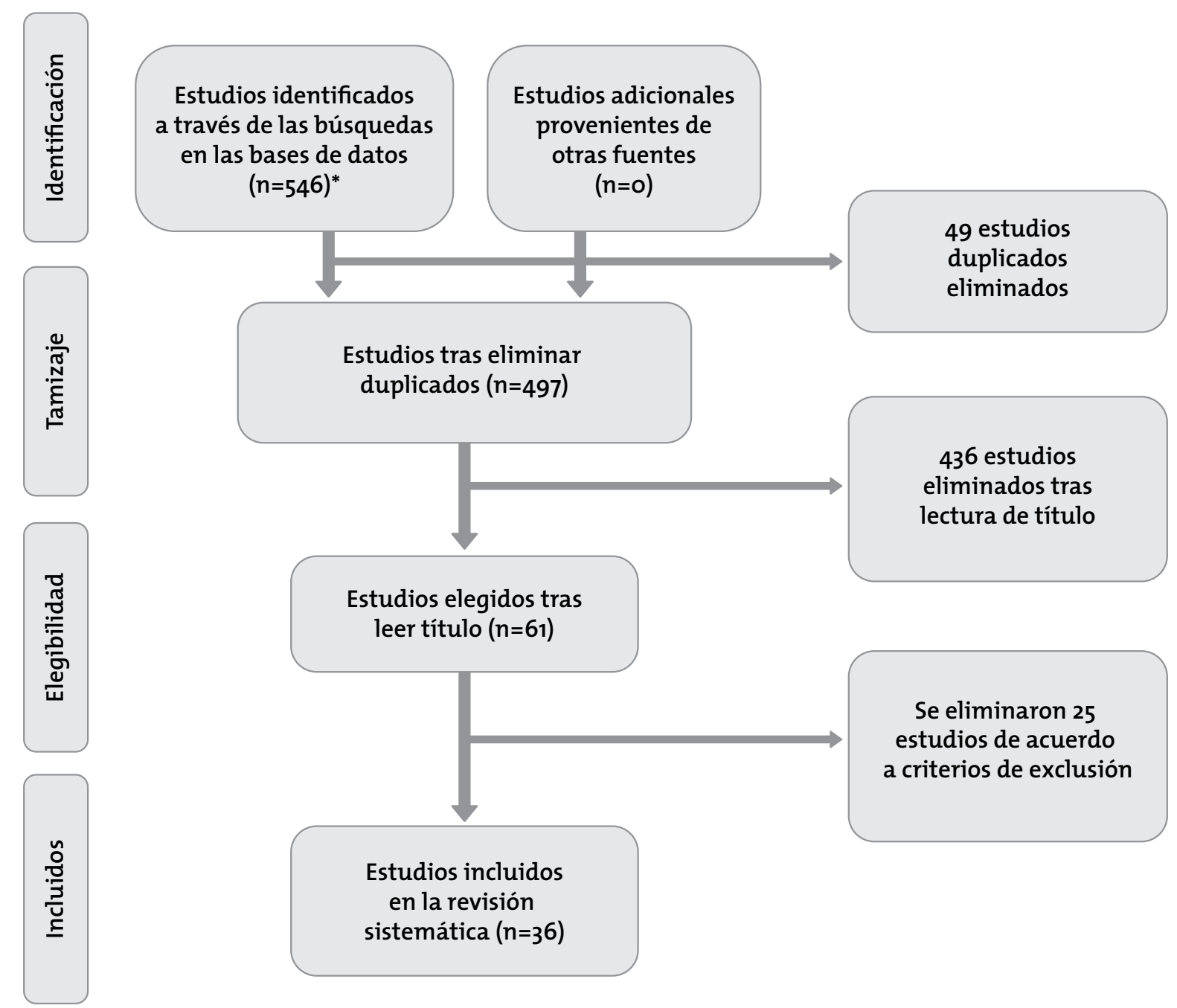

* Redaly c, 123; Dialnet, 233; Scielo, 57; Ebsco Host, fuente académica, 133. Fuente: elaboración propia. 


\section{Características de los estudios}

Los 36 estudios incluidos en esta revisión se presentan en las Tablas 1 y 2, donde se muestra la base de datos de la cual provienen, el título del artículo, los/ las autores, el año, el país, el tipo de investigación y el foco de interés. En su mayoría, los estudios provienen de México (38,9\%), Brasil (22,2\%), seguidos por Colombia $(16,7 \%)$ y Venezuela y Costa Rica $(5,6 \%)$ (Tabla 3$)$.

Tabla 1. Estudios revisados sobre representaciones sociales y percepción del CC

\begin{tabular}{|c|c|c|c|c|}
\hline Base de datos & Título & País & $\mathrm{Tl}^{*}$ & Foco de interés \\
\hline Redalyc, Scielo & $\begin{array}{l}\text { - ¿Qué piensan, dicen y hacen los jóvenes universitarios sobre el cambio climático? Un } \\
\text { estudio de representaciones sociales (González y Maldonado, 2014). }\end{array}$ & México & $\mathrm{D}$ & \multirow{8}{*}{$\begin{array}{l}\text { Estudiantes } \\
\text { universitarios }\end{array}$} \\
\hline Dialnet & $\begin{array}{l}\text { - Culturas académicas en la representación social del cambio climático (Gutiérrez, } \\
\text { 2015). }\end{array}$ & $\begin{array}{l}\text { México y } \\
\text { España }\end{array}$ & $\mathrm{D}$ & \\
\hline Redalyc, Scielo & $\begin{array}{l}\text { - Ideas sobre cambio climático y consumo energético en estudiantes de Ingeniería de } \\
\text { la Unexpo-LCM (Gómez y Moncada, 2015). }\end{array}$ & Venezuela & $\mathrm{D}$ & \\
\hline $\begin{array}{l}\text { Dialnet, Redalyc, } \\
\text { Scielo }\end{array}$ & $\begin{array}{l}\text { - Representaciones sociales del cambio climático en estudiantes de dos universidades } \\
\text { veracruzanas (Ramírez y González, 2016). }\end{array}$ & México & $\mathrm{D}$ & \\
\hline Dialnet, Redalyc & $\begin{array}{l}\text { - El uso del currículo oculto en la interpretación de cambio climático en infografías de } \\
\text { estudiantes de educación superior (Colón, 2016). }\end{array}$ & Puerto Rico & $\mathrm{D}$ & \\
\hline Dialnet & - El cambio climático: una perspectiva de género (Olmos et al., 2016). & México & $\mathrm{D}$ & \\
\hline Scielo Mx & $\begin{array}{l}\text { - El cambio climático en las representaciones sociales de los estudiantes universitarios } \\
\text { (Calixto, 2018). }\end{array}$ & México & $\mathrm{D}$ & \\
\hline Scielo Br & $\begin{array}{l}\text { - Las emociones en las representaciones sociales del cambio climático (Calixto y } \\
\text { Amigon, 2018). }\end{array}$ & México & $\mathrm{D}$ & \\
\hline $\begin{array}{l}\text { Dialnet, Redalyc, } \\
\text { Ebsco Host }\end{array}$ & $\begin{array}{l}\text { - Las representaciones sociales del cambio climático en estudiantes de educación } \\
\text { secundaria (Calixto, 2015a). }\end{array}$ & México & $\mathrm{D}$ & \multirow{2}{*}{$\begin{array}{l}\text { Estudiantes de } \\
\text { secundaria }\end{array}$} \\
\hline $\begin{array}{l}\text { Dialnet, Redalyc, } \\
\text { Scielo }\end{array}$ & $\begin{array}{l}\text { - Representaciones sociales sobre cambio climático en dos grupos de estudiantes de } \\
\text { educación secundaria de España y bachillerato de México (Bello et al., 2017). }\end{array}$ & México & $\mathrm{D}$ & \\
\hline Redalyc & $\begin{array}{l}\text { - Construcción de conocimiento y creencias epistemológicas sobre cambio climático } \\
\text { en docentes de nivel primaria. De la vulnerabilidad a la resiliencia (Cajigal et al., } \\
\text { 2016). }\end{array}$ & México & $\mathrm{D}$ & \multirow{2}{*}{ Docentes } \\
\hline Redalyc & $\begin{array}{l}\text { - Resiliencia en docentes: Una vía para mejorar capacidades de las poblaciones ante } \\
\text { inundaciones agravadas por el cambio climático (Cajigal et al., 2017). }\end{array}$ & México & $\mathrm{D}$ & \\
\hline Dialnet & $\begin{array}{l}\text { - La perspectiva del cambio climático en el departamento de Antioquia (Paniagua y } \\
\text { Hernández, 2013). }\end{array}$ & Colombia & $\mathrm{D}$ & \multirow{11}{*}{ Ciudadanos } \\
\hline $\begin{array}{l}\text { Google académico, } \\
\text { Dialnet, Scielo Co }\end{array}$ & $\begin{array}{l}\text { - Los jóvenes de la ciudad de Popayán frente al cambio climático. Un estudio desde las } \\
\text { representaciones sociales (Alvear et al., 2015). }\end{array}$ & Colombia & $\mathrm{D}$ & \\
\hline Redalyc & $\begin{array}{l}\text { - Estudio sobre la percepción social del concepto de cambio climático y su divulgación en } \\
\text { los medios de comunicación en la región de Santander-Colombia (Moreno et al., 2015). }\end{array}$ & Colombia & $\mathrm{D}$ & \\
\hline $\begin{array}{l}\text { Ebsco Host, } \\
\text { Redalyc }\end{array}$ & $\begin{array}{l}\text { - Gestión de riesgo de desastres, género y cambio climático. Percepciones sociales en } \\
\text { Yucatán, México (Soares y Murillo, 2013). }\end{array}$ & México & $\mathrm{D}$ & \\
\hline Ebscohost & - Perceptions of climate change on the Island of Providencia (Altschuler y Brownlee, 2016). & Colombia & $\mathrm{D}$ & \\
\hline Ebsco Host & $\begin{array}{l}\text { - El conocimiento, la percepción y disponibilidad para afrontar el cambio climático en } \\
\text { una población emergente, los migrantes de retorno (Corona, 2018). }\end{array}$ & México & $\mathrm{D}$ & \\
\hline Scielo $M x$ & $\begin{array}{l}\text { - Una aproximación a la representación del cambio climático en habitantes de dos } \\
\text { cuencas del estado de Veracruz, México (Maldonado et al., 2017). }\end{array}$ & México & D & \\
\hline Scielo $\mathrm{Br}$ & $\begin{array}{l}\text { - Mudanças climáticas e distribuição social da percepção de risco no Brasil (Bursztyn } \\
\text { y Eiro, 2015). }\end{array}$ & Brasil & $\mathrm{D}$ & \\
\hline Dialnet & $\begin{array}{l}\text { - Turismo, información, cambio climático: El caso del Parque Nacional Volcan Poas, } \\
\text { Costa Rica (Aguirre, 2011). }\end{array}$ & Costa Rica & D & \\
\hline $\begin{array}{c}\text { Google Academico, } \\
\text { Dialnet }\end{array}$ & $\begin{array}{l}\text { - La representación social del cambio climático: una revisión internacional (González, } \\
\text { 2012). }\end{array}$ & $\begin{array}{l}\text { Europa } \\
\text { América }\end{array}$ & Dc & \\
\hline Scielo $\mathrm{Br}$ & $\begin{array}{l}\text { - Percepção ambiental sobre as mudanças climáticas globais numa praça pública na } \\
\text { cidade do Rio de Janeiro (RJ, Brasil) (Pedrini et al., 2016). }\end{array}$ & Brasil & D & \\
\hline
\end{tabular}

*TI:Tipo de investigación; D: Descriptiva; RS: Representaciones sociales; E: ensayo; I: Intervención; Dc: Documental. Fuente: elaboración propia. 
Tabla 2. Estudios revisados sobre propuestas educativas

\begin{tabular}{|c|c|c|c|c|}
\hline Base de datos & Título & Pais & TI & Foco de interés \\
\hline Scielo Br & $\begin{array}{l}\text { - Mudanças climáticas, educação e meio ambiente: para além do } \\
\text { Conservadorismo Dinâmico (Lima y Layrargues, 2014). }\end{array}$ & Brasil & E & \multirow{3}{*}{$\begin{array}{l}\text { Educación, } \\
\text { fundamental para } \\
\text { cultura ambiental }\end{array}$} \\
\hline Redalyc & $\begin{array}{l}\text { - Educación ambiental y cambio climático. Necesidad de desarrollar y } \\
\text { fortalecer capacidades locales (Rosete, 2014). }\end{array}$ & $\begin{array}{l}\text { América Latina } \\
\text { y el Caribe }\end{array}$ & E & \\
\hline $\begin{array}{l}\text { Google } \\
\text { Academico }\end{array}$ & $\begin{array}{l}\text { - Educación ambiental en el marco de una estrategia participativa } \\
\text { para atender el cambio climático a nivel local: Experiencias en Costa } \\
\text { Rica (Valerio et al., 2016). }\end{array}$ & Costa rica & I & \\
\hline Dialnet & $\begin{array}{l}\text { - Propuesta en educación ambiental para la enseñanza del cambio } \\
\text { climático (Calixto, 2015b). }\end{array}$ & México & $\mathrm{D}$ & Uso de noticias \\
\hline Ebsco Host & $\begin{array}{l}\text { - International perspectives on the pedagogy of climate change } \\
\text { (Perkins et al., 2018). }\end{array}$ & $\begin{array}{l}\text { Brasil, China, } \\
\text { Alemania, } \\
\text { México, Arabia } \\
\text { Saudi, EE.UU. }\end{array}$ & $\mathrm{D}$ & \multirow{6}{*}{$\begin{array}{c}\text { Abordaje } \\
\text { multidimensional }\end{array}$} \\
\hline Scielo Br & $\begin{array}{l}\text { - Mudanças climáticas globais: a resposta da educação (Jacobi et al., } \\
\text { 2011). }\end{array}$ & Brasil & E & \\
\hline Scielo Br & $\begin{array}{l}\text { - Mudanças climáticas e ensino superior: a combinação entre } \\
\text { pesquisa e educação (Jacobi, 2014). }\end{array}$ & & & \\
\hline Redalyc & $\begin{array}{l}\text { - Controvérsias sobre o aquecimento global: circulação de vozes e de } \\
\text { sentidos produzidos em sala de aula (Barbosa et al., 2012). }\end{array}$ & Brasil & I & \\
\hline Scielo br & $\begin{array}{l}\text { - Análise de dissertações e teses brasileiras de Educação Ambiental: } \\
\text { compreensões elaboradas sobre o tema "mudanças climáticas" (Reis } \\
\text { y Silva, 2016). }\end{array}$ & Brasil & Dc & \\
\hline Scielo & $\begin{array}{l}\text { - As complexidades inerentes ao tema "mudanças climáticas": } \\
\text { desafios e perspectivas para o ensino de física (Reis et al., 2015). }\end{array}$ & Brasil & E & \\
\hline Dialnet & $\begin{array}{l}\text { - La importancia de la participación ciudadana a través de la } \\
\text { educación ambiental para la mitigación del cambio climático a nivel } \\
\text { local (Alegre, 2010). }\end{array}$ & $\begin{array}{l}\text { Argentina, } \\
\text { Colombia }\end{array}$ & I & \multirow{4}{*}{$\begin{array}{l}\text { Participación } \\
\text { clave para } \\
\text { educación para } \\
\quad \text { el CC }\end{array}$} \\
\hline Scielo & $\begin{array}{l}\text { - Programa de educación ambiental sobre el cambio climático en la } \\
\text { educación formal y no formal (González, 2016). }\end{array}$ & Venezuela & $\mathrm{D}$ & \\
\hline Dialnet & $\begin{array}{l}\text { - Cambio climático, gestión y educación ambiental urbana: análisis } \\
\text { geopolítico. Estudio de caso: Complejo de humedales en la localidad } \\
\text { de 11-Suba, Bogotá (Pinilla y Puertas, 2017). }\end{array}$ & Colombia & I & \\
\hline $\begin{array}{l}\text { Google } \\
\text { Académico } \\
\text { Scielo } \mathrm{Br}\end{array}$ & $\begin{array}{l}\text { - Experiencias en la aplicación de la educación ambiental como } \\
\text { herramienta para la adaptación al cambio climático en espacios } \\
\text { comunitarios, en Holguín-Cuba (Gallardo, 2014). }\end{array}$ & Cuba & I & \\
\hline
\end{tabular}

* TI:Tipo de investigación; D: Descriptiva; RS: Representaciones sociales; E: Ensayo; I: Intervención; Dc: Documental. Fuente: elaboración propia. 
Tabla 3. Número de publicaciones por países

\begin{tabular}{|l|c|c|}
\hline \multicolumn{1}{|c|}{ País } & No. articulos & Porcentaje \\
\hline América Latina y el Caribe & 1 & $2,8 \%$ \\
\hline Brasil & 8 & $22,2 \%$ \\
\hline Brasil, China, Alemania, México, Arabia Saudí, EE.UU. & 1 & $2,8 \%$ \\
\hline Colombia & 6 & $16,7 \%$ \\
\hline Costa Rica & 2 & $5,6 \%$ \\
\hline Cuba & 1 & $2,8 \%$ \\
\hline México & 14 & $38,9 \%$ \\
\hline Puerto Rico & 1 & $2,8 \%$ \\
\hline Venezuela & 2 & $5,6 \%$ \\
\hline
\end{tabular}

Fuente: elaboración propia.

Desde el punto de vista del tipo de estudio, se caracterizan por ser principalmente investigaciones de tipo descriptivo (69,4\%), seguidas de ensayos teóricos $(13,9 \%)$, de intervención $(13,9 \%)$ e investigaciones de tipo documental (2,8\%) (Tabla 4). Los 36 estudios seleccionados en esta revisión fueron clasificados en dos áreas de exploración: estudios de percepción y representaciones sociales sobre CC: $63,9 \%$ y propuestas educativas $36,1 \%$ (Tabla 5 ). A continuación, se describen los principales hallazgos de cada una de estas áreas en las que se agrupan las investigaciones con sus respectivos focos de interés.

Tabla 4. Tipo de investigaciones

\begin{tabular}{|c|c|c|}
\hline Tipo de investigación & No. articulos & Porcentaje \\
\hline Descriptiva & 25 & $69,4 \%$ \\
\hline Ensayo teórico & 5 & $13,9 \%$ \\
\hline Intervención & 5 & $13,9 \%$ \\
\hline Documental & 1 & $2,8 \%$ \\
\hline
\end{tabular}

Fuente: elaboración propia. 
Tabla 5. Número de artículos por áreas exploradas y focos de interés

\begin{tabular}{|c|c|c|c|}
\hline Área explorada & Focos de interés & No. articulos & Porcentaje \\
\hline \multirow[t]{5}{*}{ Estudios de percepción y RS del CC } & & 23 & $63,9 \%$ \\
\hline & Estudiantes universitarios & 7 & \\
\hline & Estudiantes de secundaria & 2 & \\
\hline & Percepción de docentes & 2 & \\
\hline & Percepción de ciudadanos & 12 & \\
\hline \multirow[t]{5}{*}{ Propuesta educativa } & & 13 & $36,1 \%$ \\
\hline & Educación, fundamental para la cultura ambiental & 3 & \\
\hline & Uso de noticias & 1 & \\
\hline & Abordaje multidimensional & 5 & \\
\hline & $\begin{array}{l}\text { Participación, clave en educación para la } \\
\text { adaptación y mitigación del CC }\end{array}$ & 4 & \\
\hline
\end{tabular}

Fuente: elaboración propia.

\section{Estudios de percepción y representaciones sociales del CC}

El 60,5\% de los estudios encontrados en esta revisión abordan el tema del CC a partir de qué piensan o qué percepciones tienen las personas acerca del fenómeno. Este tipo de estudios aportan un marco referencial, con el fin de que se pueda educar mejor, para lo cual es indispensable entender lo que el público piensa, de tal manera que se comprometa, actúe promoviendo cambios personales y colectivos y se involucre en el cambio de normas sociales, políticas y culturales. A continuación, se mostrarán los estudios de percepción y representaciones sociales (RS) del CC realizados con estudiantes universitarios y de educación secundaria, docentes y ciudadanos.

\section{Representaciones sociales sobre el CC en estudiantes universitarios:}

Las investigaciones ubicadas en este foco de interés pretenden identificar las percepciones que tienen los estudiantes acerca del fenómeno y los factores que pueden incidir en las mismas. De los 23 estudios, 10 utilizan población joven como objeto de estudio. Uno de los factores importantes que se ha logrado identificar como elemento relevante en las representaciones sociales -captado en estudiantes de la Universidad Pedagógica de México-es la emoción, la cual resultaría interesante estudiar desde la óptica de la educación para el CC (Calixto, 2018; Calixto y Amigón, 2018). También se encontró que los estudiantes de la universidad veracruzana piensan que el país tiene una responsabilidad mayor que el individuo en lo que toca a las causas del CC. Esta creencia afecta la acción ciudadana para hacer frente al CC (Gutiérrez, 2015). Y, contrario a lo que se podría pensar, la cultura académica no es un factor determinante en las representaciones sociales de CC relativas a las causas, consecuencias y medidas de mitigación. La inercia de la cultura común se sobrepone a y persiste sobre la cultura científica, incluso en estudiantes de carreras marcadamente científicas ante cuestiones científicamente falsas. Por ejemplo, sobre la idea de que la lluvia ácida es una de las causas del CC, 69,5\% de la muestra piensa que esta afirmación es probable o totalmente verdadera (Gutiérrez, 2015). El otro estudio evalúa también las percepciones en estudiantes de tres universidades mexicanas: Universidad Autónoma de Baja Califor- 
nia Sur, el Instituto Tecnológico de La Paz y la Universidad Mundial (Olmos et al., 2016). Se observa en esta última que todos los estudiantes tienen información confusa acerca del fenómeno y que hay diferencias entre hombres y mujeres sobre las causas del CC. Las mujeres opinan que se debe al agujero en la capa de ozono, mientras que los hombres aluden a la contaminación del aire; sin embargo, ambos géneros concuerdan en que el principal impacto es la sequía y escasez de agua y el nivel de afectación es alto para toda la población y las generaciones futuras. Las acciones que los universitarios consideran relevantes ante el problema son las de apagar las luces y reciclar; sin embargo, esta última acción solo la hace el $7 \%$ de los hombres y el $8 \%$ de las mujeres, aunque más del $80 \%$ admite que la ciudadanía debe tomar acciones frente al CC. Las mujeres opinan que el gobierno debe tomar medidas prioritarias sobre el CC y los hombres priorizan la educación. Se concluye que hay desinformación y que los universitarios ven el problema como algo alejado de su vida cotidiana (Olmos et al., 2016).

Ante estos resultados, Gutiérrez (2015) piensa que el conocimiento de estudiantes universitarios sobre el tema o es insuficiente o la información que reciben de los distintos medios, materiales y programas, en algunos casos, puede que no sea del todo correcta, mientras que en otros, de manera personal, cada uno reelabora y transforma esa información para comprender qué está sucediendo en su país o entorno. En el estudio realizado por González y Maldonado (2014) con estudiantes de licenciatura y posgrado de la misma universidad, se encontró que la información científica transmitida por diversas fuentes, científicas y populares, no es suficiente para generar cambios de comportamiento en los jóvenes, orientados a mitigar el CC. Se recomiendan nuevas estrategias de comunicación y de educación ambiental dirigidas a generar conductas ambientalmente sustentables.

Es frecuente encontrar en estos estudios conceptos erróneos relacionados con el CC (Colón, 2016) y ausencia de información en aspectos relevantes del tema, como sumideros de carbono, comercio de emisiones y protocolo de Kioto (Calixto, 2018). También, según el estudio de Gómez y Moncada (2015), realizado con estudiantes del curso Química de la UnexpoLCM, se encontró que existía poca información sobre el CC en el grupo en estudio. La información existente provenía de vivencias de su entorno y se desconocía la relación del CC con el consumo energético, en especial el vinculado al transporte. De este diagnóstico los autores proponen la incorporación de una unidad didáctica sobre el tema. En la investigación realizada por Ramírez y Gonzalez (2016) para comparar la incidencia del contexto en las representaciones sociales de estudiantes de una universidad pública y una privada en la ciudad de Xalapa (México), se determinó que no hay incidencia de este factor en las representaciones sociales de los estudiantes y que hay coincidencia en la información que manejan sobre CC, en ambas universidades.

\section{Representaciones sociales sobre el CC en estudiantes de secundaria:}

Las representaciones sociales (RS) resultan relevantes para comprender los sentidos y los significados que los sujetos le confieren a determinado objeto o fenómeno, como el CC, y para comprender la postura que asumen ante los problemas ambientales. En este foco de interés se ubican estudios que evalúan las RS en estudiantes de bachillerato. Uno fue realizado en México, donde las RS del CC fueron dominadas por elementos naturales: biológicos, fisicoquímicos, atmosféricos, hidrológicos, entre otros, dejando en un segundo término elementos sociales y culturales. Se resalta la importancia de construir estrategias educativas que incidan en una mejor comprensión de los problemas ambientales desde la educación básica (Calixto, 2015a). Otro fue realizado en México y España, donde ambos grupos de estudiantes privilegian información sobre aspectos biofísicos del CC. Esta percepción común está estrechamente relacionada con procesos de difusión 
globalizados del fenómeno. También, en porcentajes similares, ambos grupos expresan una visión lejana acerca de sus afectaciones. En la mayoría de los casos reconocen la influencia antrópica en el CC y las consecuencias en el medio natural. Asimismo, se advierte la influencia de la información proveniente de los medios de comunicación sobre el fenómeno (Bello et al., 2017).

\section{Percepciones de docentes:}

En este foco se encuentran dos estudios que resaltan la labor docente como elemento clave en el proceso de educación para el CC. En el primero se evalúa el tipo de perspectivas del conocimiento que tienen acerca del CC, así como su percepción de los desastres, en los docentes de educación primaria del municipio de Juchique de Ferrer, Veracruz (México), y su papel en los espacios escolares (Cajigal et al., 2016); en el otro se evalúa la percepción de los docentes de bachillerato de la comunidades de Tlacotalpan, Cotaxtla y La Antigua en el estado de Veracruz, y los proponen como elementos clave para desarrollar resiliencia en las comunidades, debido a que resultan ser actores importantes en la comunidad y, por tanto, promotores de acciones que propicien resiliencia.

\section{Percepciones de ciudadanos:}

De las 12 investigaciones que evalúan las percepciones de los ciudadanos sobre el CC, cuatro fueron realizadas en Colombia. La primera, adelantada en la isla de Providencia, indica que los ciudadanos reconocen cambios en su entorno, pero dan explicaciones locales, no globales, como el calentamiento. Sin embargo, las percepciones de muchos de los residentes sobre el CC se alinean con los pensamientos prevalecientes de los científicos del clima (Altschuler y Brownlee, 2016). La segunda se realizó en el departamento de Antioquia y los resultados indican que existe poco compromiso para la prevención del CC y poco interés de los gobernantes para expedir y/o implementar normas que permitan la adaptación al CC. Los encuestados coinciden en que los sectores más vulnerables al CC, en lo económico, son las unidades productivas agrícolas y pecuarias y el sector energético; en lo social, las afectaciones a comunidades generadas por condiciones geomorfológicas; y en lo ambiental, la pérdida de biodiversidad (Paniagua y Hernández, 2013). La tercera investigación se realizó en Popayán y encontró que los ciudadanos entienden el proceso del CC, sus causas y consecuencias, y que esta información proviene en su mayor parte de los medios de comunicación, como Internet y TV, pero lo identifican más como una problemática global que local. En el ámbito local, para ellos son más representativos la contaminación y algunos problemas sociales (Alvear et al., 2017). Y la cuarta se realizó en la ciudad de Bucaramanga, donde se encontró que hay pocas (53\%) piezas informativas sobre CC en los medios, pero que se trata de noticias entendibles (66\%). Existe una percepción clara (84\%) de que el clima del planeta está cambiando por causas provocadas principalmente por el hombre. La mayoría (87\%) manifiesta preocupación por el planeta y la necesidad de estar informados (84\%) sobre estos temas a través de los medios de comunicación. Pero no es tan evidente que la ciudadanía conozca conceptos clave como el de la huella ecológica o se preocupe por el reciclaje ( $44 \%$ y $63 \%$ respectivamente), para implementar soluciones (Moreno et al., 2015).

Finalmente, los estudios realizados en Colombia concluyen en la importancia de la educación como principal instrumento y herramienta de conocimiento y acercamiento al problema del CC y la necesidad de diseñar estrategias de comunicación y educación ambiental orientadas a una participación activa de la población en los procesos de adaptación y mitigación al CC, con la idea de lograr cambios de actitudes y de comportamientos sociales para contribuir a frenar el CC.

Otros estudios fueron realizados en diferentes países. En México se evalúa la percepción de los migrantes de retorno. Se encontró que la mayoría de ellos se enteraron del CC en los Estados Unidos, te- 
nían un conocimiento incipiente del mismo y una gran disposición para participar en acciones de adaptación y mitigación. Estos ciudadanos podrían desempeñar un papel protagónico, si fueran incorporados como agentes promotores del desarrollo regional sustentable (Corona, 2018). Otro estudio evalúa las RS de la población adulta de las cuencas de los ríos Jamapa y Pixquiac (México), cuya población admite la existencia del CC e identifica problemas ambientales locales, varios de ellos relacionados con el fenómeno en lo que toca a sus causas o consecuencias. Atribuyen el CC a las acciones humanas y son conscientes de que el CC agrava problemas como pobreza, migraciones, enfermedades, desnutrición y hambrunas. Es evidente la ausencia de acciones institucionales de mitigación y adaptación. La televisión es el medio por el que la mayoría se informa sobre el tema y las autoridades responsables tienen poca credibilidad entre la población (Maldonado et al., 2017). En estudio realizado por Soares y Murillo (2015) se evalúan tres comunidades del estado de Hunucmá (México) en la cual se evidencia una fuerte relación del CC con los huracanes, hay diferencia entre hombres y mujeres en lo que saben acerca de qué es el cambio climático. A la cual los hombres responden si en un 70\% y las mujeres en un $52 \%$. En cuanto a si creen en la existencia del fenómeno no hubo diferencias entre géneros.

En Brasil se evalúa la percepción sobre el CC de ciudadanos que asisten al evento "Desapegue" ocurrido en la plaza Edmundo Rego en la ciudad de Río de Janeiro, en donde se confirmó la hipótesis de que ese público tendría información básica conceptual adecuada sobre educación ambiental y CC (Pedrini et al., 2016). En otra investigación para todo el país se mide la percepción de riesgo del CC y se encontró que existe una creencia generalizada de que hay riesgo para la población (Bursztyn y Eiró, 2015).

Por último, en Costa Rica se evaluó la percepción de los visitantes del Parque Nacional Volcán Poas (abril de 2008) en relación con sus actitudes frente al CC y qué fuentes de información usan respecto a este tema. Los visitantes del parque provenían de Costa Rica, Estados Unidos y Europa, con edades entre 30 y 50 años. Frente a la importancia del CC como problema hoy, y en una escala de o a 5, para todos estuvo en 2, y en cincuenta años, en 4; sin embargo, los costarricenses parecen estar más preocupados por el problema que los demás. Los europeos y norteamericanos indicaron que la deforestación es la principal causa del CC, seguida por las emisiones de autos. Los costarricenses obtuvieron su información sobre el CC en la televisión, los norteamericanos en la televisión e internet y los europeos en televisión, internet y la academia. El estudio concluye, de acuerdo con el análisis inferencial de datos, que hay una fuerte influencia de los medios de comunicación en las actitudes, conocimientos y percepciones del problema del CC (Aguirre, 2011). Se han detectado confusiones bastante generalizadas a nivel mundial entre el CC y otros problemas ambientales (capa estratosférica de ozono, lluvia ácida, contaminación atmosférica, etc.), así como entre el clima y el tiempo. Este tipo de confusiones entorpecen las representaciones apropiadas sobre las causas del fenómeno y causan confusiones entre el clima y el tiempo atmosférico (González, 2012).

\section{Propuestas educativas}

En esta categoría se encuentran 13 investigaciones relacionadas con propuestas educativas para incidir en el comportamiento de las personas.

\section{Educación, fundamental para cultura ambiental:}

Tres (3) de las 13 investigaciones hablan de que la educación es fundamental para lograr una nueva cultura ambiental en la población, así como para desarrollar y fortalecer las capacidades locales necesarias para el cumplimiento de las acciones de mitigación y adaptación al CC impulsadas por las instituciones gubernamentales y de la sociedad civil (Rosete, 2014). También remarcan que la educación ayuda a renovar la comprensión del problema y la acción de los individuos, de las instituciones y de 
los agentes públicos y privados involucrados con el tema (Lima y Layrargues, 2014). La educación se convierte entonces en un eje clave para trabajar el tema de CC a nivel local, donde las comunidades y los actores sociales ambientalmente educados pueden convertirse en sujetos del desarrollo, participantes en la toma de decisiones y en la definición de políticas públicas para sus territorios (Valerio et al., 2016)

\section{Uso de noticias:}

En el estudio realizado por Calixto (2015b) se plantea el uso de noticias, tomadas directamente de la televisión, con el fin de que los estudiantes, a través de preguntas reflexivas, piensen acerca del consumismo y la manera como se relaciona con el CC, los estándares sociales y el estilo de vida. Esta propuesta implica el uso de procesos complejos, donde el estudiante se involucre en situaciones para saber cómo actuar en determinados contextos, realizar actividades y/o resolver problemas desde la perspectiva del procesamiento metacognitivo.

\section{Abordaje multidimensional:}

En este foco de interés se ubican diferentes investigaciones en las que se plantea que la educación debe abordar el CC desde una perspectiva multidimensional donde se involucren: los contextos culturales y pedagogías de participación (Perkins et al., 2018); la capacitación social con base cooperativa próxima al pensamiento crítico y a la habilidad para la resolución de problemas (Jacobi, 2014); la elaboración de materiales didácticos con conocimientos interdisciplinares acumulados sobre el clima y los cambios globales (Jacobi et al., 2011); las complejidades del cambio, debido a que muchos discursos elaborados por una parte de los medios en relación con ese tema contienen equívocos conceptuales y presentan enfoques sensacionalistas, hecho que acaba por privilegiar una visión exclusivamente catastrófica y determinista relativa al fenómeno (Reis et al., 2015); y la utilización de problemas sociocientíficos y la controversia sobre el tema del CC (Barbosa et al., 2015; Reis y Silva, 2016).

\section{Participación, clave en educación para la adaptación y mitigación del CC:}

Otro foco de interés que resalta en las investigaciones de educación para el CC, específicamente dentro de las propuestas educativas, lo constituyen las investigaciones donde se destaca que la participación es un elemento clave en la educación para el CC. Se proponen estrategias para capacitar comunidades sobre CC, contemplando los modos de participación comunitaria y desarrollando cronogramas de acciones puntuales, para generar conciencia y que se vea la trasformación de sus comunidades a futuro (Alegre, 2010; Gallardo, 2014). El hecho de participar en actividades evidencia un cambio de actitud en cuanto a los conocimientos adquiridos, mejor comprensión problemas que se ocasionan en el ambiente y en la salud de los seres vivos y la disposición e interés por aplicar medidas y desarrollar acciones a favor del clima (González, 2016).

Se propone también una ruta pedagógica basada en comunidades y ecosistemas para la adaptación y mitigación del CC en el circuito ecosistémico entre los humedales Torca-Guaymaral y Córdoba, en Bogotá, donde intervienen diferentes actores: instituciones gubernamentales, educativas y comunidad en general, así: 1) toma de conciencia, 2) adquirir conocimientos, 3) cambio de actitudes, 4) fomentar aptitudes, 5) capacidad de evaluación, 6) participación (Pinilla y Puertas, 2017).

\section{Conclusiones}

Las investigaciones en educación para el CC registradas en esta revisión muestran que en la región latinoamericana se han realizado principalmente estudios sobre percepción y representaciones sociales y sobre la manera de abordar el tema del CC en el campo educativo. Entre los estudios de percepción se han hecho exploraciones en diferentes países y grupos poblacionales, como jóvenes universitarios, jóvenes de educación secundaria, docentes y ciudadanos; sin embargo, la mayor parte de ellos develan conceptos errados sobre el fenómeno, confusiones 
entre el CC y otros problemas ambientales (capa estratosférica de ozono, lluvia ácida, contaminación atmosférica, fenómenos atmosféricos como huracanes), así como entre el clima y el tiempo. También se ve el fenómeno como algo lejano. Esto dificulta la toma de acciones adecuadas y oportunas por parte de la sociedad. En países como México y Brasil se ha dado importancia al estudio de las RS en población joven, principalmente en estudiantes de segundaria y universitarios. En Colombia los estudios de percepción se han aplicado a ciudadanos de varios lugares, pero no se ha hecho énfasis en lo que piensan los jóvenes, quienes representan el futuro del país y con quienes trabajar podría resultar promisorio para emprender acciones relevantes que contribuyan a la mitigación y adaptación al fenómeno.

Entre las propuestas educativas están las que plantean usar las noticias de la televisión como herramienta para la reflexión; las que proponen que la educación para la mitigación y la adaptación al CC debe ser una apuesta multidisciplinar donde se traten todas las temáticas que rodean el fenómeno, desde diferentes disciplinas, teniendo en cuenta complejidades y aspectos socioculturales; las que proponen que la participación es fundamental en la toma de conciencia por parte de las comunidades; $y$, por último, las que se enfocan en la educación a pequeñas y medianas empresas, quienes sería claves en la mitigación del fenómeno. Estas propuestas educativas son en su mayoría de carácter teórico.

Los abordajes metodológicos que caracterizan las investigaciones publicadas han consistido en gran parte en encuestas de percepción. Se echan de menos diseños preexperimentales, cuasiexperimentales y experimentales. En este sentido, son muy pocos los estudios que ponen a prueba alguna variable o didáctica que muestre, en especial, algún impacto en la transformación de las RS o directamente sobre los comportamientos de las personas para mitigar los efectos del CC o adaptarse a las nuevas condiciones que este impondrá. Al predominar los trabajos descriptivos, se desconoce el impacto que tienen las distintas aproximaciones para la enseñanza del CC, lo cual, de alguna manera, está en concordancia con los hallazgos encontrados en las revisiones sobre educación ambiental internacional de Chawla y Derr (2012) y Medina y Páramo (2014), para América Latina. En ellos, llama la atención que la mayoría de los trabajos no evalúan la efectividad de las intervenciones sobre el cambio directo del comportamiento y que aún son predominantes los artículos de carácter descriptivo, aunque se reconoce que son la línea de base que abre las puertas a estudios de intervención o experimentales.

Es necesario entonces aumentar y afinar el conocimiento básico y aplicado sobre la percepción social del CC en América Latina, para poder diseñar, aplicar y legitimar acciones educativas en el marco de las políticas de respuesta a la crisis climática. Esta revisión sistemática muestra la necesidad de llevar a cabo estudios que generen impacto en la formación de las personas y evalúen las incidencias de estas experiencias educativas en la mitigación y adaptación al cambio climático. 


\section{Referencias}

Aguirre-González, J. A. (2011). Turismo, información, cambio climático: El caso del Parque Nacional Volcán Poas, Costa Rica. Pasos. Revista de Turismo y Patrimonio Cultural, 9(4), 519-530. http://www.pasosonline.org/ Publicados/9411/PSO411_03.pdf

Alegre, S. I. (2010). La importancia de la participación ciudadana a través de la educación ambiental para la mitigación del cambio climático a nivel local. Delos. Desarrollo Local Sostenible, 3(7), 1-11 http://www.eumed. net/rev/delos/o7/sia.htm

Altschuler, B. y Brownlee, M. (2016). Perceptions of climate change on the Island of Providencia. Local Environment, 21(5), 615-635. https://doi.org/10.1080/13549839.2015.1004165

Alvear-Narváez, N. L., Ceballos-Sarria, V. E.y Urbano-Bolaños, M. (2015). Los jóvenes de la ciudad de Popayán frente al cambio climático. Un estudio desde las representaciones sociales. Revista Ingenierías, Universidad de Medellin, 14(26), 43-56. http://www.scielo.org.co/pdf/rium/v14n26/v14n26ao4.pdf

Anderson, A. (2012). Climate change education for mitigation and adaptation. Journal of Education for Sustainable Development, 6(2), 191-206. https://doi.org/10.1177/0973408212475199

Barbosa, L., Lima, M. y Machado, A. (2012). Controvérsias sobre o aquecimento global: circulação de vozes e de sentidos produzidos em sala de aula. Ensaio Pesquisa em Educação em Ciências, 14(1), 113-130. https://doi. org/10.1590/1983-21172012140108

Bello-Benavides, L. O., Meira, P. y González-Gaudiano, E. J. (2017). Representaciones sociales sobre cambio climático en dos grupos de estudiantes de educación secundaria de España y bachillerato de México. Revista Mexicana de Investigación Educativa, 22(73), 505-532. http://www.scielo.org.mx/scielo.php?script=sci_abs tract\&pid=S1405-66662017000200505

Boussalis, C.y Coan, T. (2016). Text-mining the signals of climate change doubt. Global Environmental Change, 36, 89-100. https://doi.org/10.1016/j.gloenvcha.2015.12.001

Bursztyn, M. y Eiró, F. (2015). Mudanças climáticas e distribuição social da percepção de risco no Brasil. Sociedade e Estado, 30(2), 471-493. https://doi.org/10.1590/S0102-699220150002000010

Cajigal-Molina, E., Maldonado-González, A. L y González-Gaudiano, E. (2016). Construcción de conocimiento y creencias epistemológicas sobre cambio climático en docentes de nivel primaria. De la vulnerabilidad a la resiliencia. Revista Interamericana de Educación de Adultos, 38(2), 52-76. https://www.redalyc.org/articulo. oa?id $=457546143004$

Cajigal-Molina, E., Maldonado-González, A. L y González-Gaudiano, E. (2017). Resiliencia en docentes: Una vía para mejorar capacidades de las poblaciones ante inundaciones agravadas por el cambio climático. Diálogo Educacional, 17(55), 1445-1464. https://www.redalyc.org/pdf/1891/189154958002.pdf

Calixto, R. (2015a). Las representaciones sociales del CC en estudiantes de educación secundaria. Rexe. Revista de Estudios y Experiencias en Educación, 14(27), 15-32. https://dialnet.unirioja.es/descarga/articulo/5287422.pdf 
Calixto, R. (2018). El CC en las representaciones sociales de los estudiantes universitarios. Revista Electrónica de Investigación Educativa, 2O(1),122-132. https://doi.org/10.24320/redie.2018.20.1.1443

Calixto, R.y Amigón, E. (2018). Las emociones en las representaciones sociales del cambio climático. Educar em Revista, 34(68), 217-233. https://doi.org/10.1590/0104-4060.55684

Calixto, R. (2015b). Propuesta en educación ambiental para la enseñanza del CC. Diálogos Educativos, 29, 54-68. https://dialnet.unirioja.es/descarga/articulo/5159509.pdf

Cecchini, S., Espíndola, E., Filgueira, F., Hernández D. y Martínez R. (2012). Vulnerabilidad de la estructura social en América Latina: medición y políticas públicas. Revista Internacional de Estadistica y Geografía, 3(2), 32-45. https://rde.inegi.org.mx/index.php/2012/05/og/vulnerabilidad-de-la-estructura-social-en-america-latina-medicion-y-politicas-publicas/

Cepal (2014). Estudio Económico de América Latina y el Caribe 2014: desafíos para la sostenibilidad del crecimiento en un nuevo contexto externo. [Portal web] https://www.cepal.org/es/publicaciones/36970estudio-economico-america-latina-caribe-2014-desafios-la-sostenibilidad

Cepal y Unión Europea (2017). Síntesis de políticas públicas sobre cambio climático. Medidas de mitigación y adaptación al cambio climático en América Latina y el Caribe. [Documento web] https://www.cepal.org/ sites/default/files/news/files/sintesis_pp_cc_medidas_de_mitigacion_y_adaptacion.pdf

Chawla, L.y Derr, V. (2012). The development of conservation behaviors in childhood and youth. En S. Clayton (ed), The Oxford handbook of environmental and conservative psychology (pp. 527-555). Oxford University Press.

Chen, X. (2011). Why do people misunderstand climate change? Heuristics, mental models and ontological assumptions. Climatic Change, 108(1-2), 31-46. https://doi.org/10.1007/s10584-010-0013-5

Choi, S., Niyogi D., Shepardson D. y Charusombat U. (2010). Do earth and environmental science textbooks promote middle and high school students' conceptual development about climate change? Textbooks' consideration of students' misconceptions. Bulletin of the American Meteorological Society, 91(7), 889-898. https://doi.org/10.1175/2009BAMS2625.1

Clayton, S., Devine-Wright, P., Stern, P. C., Whitmarsh, L., Carrico, A., Steg, L., ... Bonnes, M. (2015). Psychological research and global climate change. Nature Climate Change, 5(7), 640-646. https://doi.org/10.1038/nclimate2622

Colón-Ortiz, A. (2016). El uso del currículo oculto en la interpretación de cambio climático en infografías de estudiantes de educación superior. Alteridad, 11(2), 182. https://doi.org/10.17163/alt.v11n2.2016.04

Corona-Jiménez, M. Á. (2018). El conocimiento, la percepción y disponibilidad para afrontar el cambio climático en una población emergente, los migrantes de retorno. Estudios Sociales: Revista de Investigación Cientifica, 28(52), 1-28. https://doi.org/10.24836/es.v28i52.578

Duarte Cueva, F. (2014). Efectos del cambio climático en la economía, el comercio internacional y la estrategia empresarial. Contabilidad y Negocios, 9(18), 75-98. http://revistas.pucp.edu.pe/index.php/contabilidadyNegocios/article/view/11613 
Gallardo-Milanés, O. (2014). Experiencias en la aplicación de la educación ambiental como herramienta para la adaptación al CC en espacios comunitarios, en Holguín-Cuba. Sociedad y Natureza, 26(2), 261-270. https:// doi.org/10.1590/1982-451320140205

Gómez Blanco, A. y Moncada, J. (2015). Ideas sobre cambio climático y consumo energético en estudiantes de Ingeniería de la Unexpo-LCM. Revista de Investigación, 39(85), 93-113. http://ve.scielo.org/scielo. php?script=sci_arttext\&pid=S1010-29142015000200006

González-Gaudiano, E. (2012). La representación social del cambio climático. Una revisión internacional. Revista Mexicana de Investigación Educativa, 17(55), 1035-1062. http://www.scielo.org.mx/scielo.php?script=sci_ar ttext\&pid=S1405-66662012000400003

González-Gaudiano, E. y Maldonado-González, A. (2014). ¿Qué piensan, dicen y hacen los jóvenes universitarios sobre el cambio climático? Un estudio de representaciones sociales. Educar em Revista, 3, 35-55. https:// www.scielo.br/pdf/er/nspez/ao4nspe3.pdf

González-Ordóñez, A. (2016). Programa de educación ambiental sobre el cambio climático en la educación formal y no formal. Revista Universidad y Sociedad, 8(3), 99-107. http://scielo.sld.cu/scielo.php?script=sci_ arttext\&pid=S2218-36202016000300013\&lng=es\&tlng=es

Gutiérrez Pérez, J. (2015). Culturas académicas en la representación social del CC. Investigar con y para la Sociedad, 1, 211-222. https://dialnet.unirioja.es/servlet/articulo?codigo=5142054

Heras, F. (2015). La educación en tiempos de cambio climático: facilitar el aprendizaje para construir una cultura de cuidado del clima. Mètode. Revista de Difusión de la Investigación, 85, 1-8. https://www.miteco.gob.es/ es/ceneam/articulos-de-opinion/2016-04-heras-hernandez_tcm30-70525.pdf

IDEAM, PNUD, MADS, DNP, Cancillería (2017). Acciones de educación, formación y sensibilización de públicos en cambio climático en Colombia. Tercera comunicación nacional de cambio climático. Ideam, PNUD, MADS, DNP, Cancillería, FMAM. Bogotá D.C., Colombia. http://documentacion.ideam.gov.co/openbiblio/ bvirtual/O23737/ACCIONES_EDUCACION_CC.pdf

IPCC - Intergovernmental Panel on Climate Change (2007). AR4 Climate Change 2007: Synthesis report. Contribution of working groups I, II, III to the Fourth Assessment Report of the International Panel on Climate Change. R. K. Pachauri y A. Reisinger (eds.) IPCC. https://www.ipcc.ch/report/ar4/syr/

IPCC - Intergovernmental Panel on Climate Change (2014). Cambio climático 2014: Informe de sintesis. Contribución de los Grupos de trabajo I, II y III al Quinto Informe de Evaluación del Grupo Intergubernamental de Expertos sobre el Cambio Climático. R. K. Pachauri y L. A. Meyer, eds. (pp.127-141). IPCC. https://www.ipcc.ch/ site/assets/uploads/2018/O2/SYR_AR5_FINAL_full_es.pdf

IPCC - Intergovernmental Panel on Climate Change (2018). Impacts of $1.5^{\circ} \mathrm{C}$ Global Warming on Natural and Human Systems. En Masson-Delmotte, V., P. Zhai, H.-O. Pörtner, D. Roberts, J. Skea, ... T. Waterfield (eds.), Global Warming of $1.5^{\circ} \mathrm{C}$. An IPCC Special Report on the impacts of global warming of $1.5^{\circ} \mathrm{C}$ above pre-industrial 
levels and related global greenhouse gas emission pathways, in the context of strengthening the global response to the threat of climate change, sustainable development, and efforts to eradicate poverty. En Prensa. https://www.ipcc.ch/sr15/

Jacobi, P., Guerra, A., Sulaiman, S.y Tiago N. (2011). Mudanças climáticas globais: a resposta da educação. Revista Brasileña de Educación, 16(46), 35-148. https://www.scielo.br/pdf/rbedu/v16n46/v16n46ao8.pdf

Jacobi, P. R. (2014). Mudanças climáticas e ensino superior: a combinação entre pesquisa e educação. Educação em Revista,3 (núm. esp.), 57-72. https://doi.org/10.1590/0104-4060.38107

Keller, L., Stotter, J., Oberrauch, A., Kuthe, A., Korfgen, A. y Hufner, K. (2019). Changing climate change education: Exploring moderate constructivist and transdisciplinary approaches through the research-education cooperation k.i.d.Z.21. Gaia - Ecological Perspectives for Science and Society, 28(1), 35. https://go.gale.com/ps/ anonymous?id=GALE\%7CA586470064\&sid=googleScholar\&v=2.1\&it=r\&linkaccess=abs\&issn=0940555 $O \& p=A O N E \& S W=W$

Leiserowitz, A., Smith, N. y Marlon, J. (2011). American teens'knowledge of climate change. Yale Project on Climate Change Communication. https://climatecommunication.yale.edu/wp-content/uploads/2016/02/2011_04_ American-Teens\%E2\%80\%99-Knowledge-of-Climate-Change.pdf

Lima, G. y Layrargues, P. (2014). Mudanças climáticas, educação e meio ambiente: para além do Conservadorismo Dinâmico. Educação em Revista, 3,73-88. http://www.scielo.br/pdf/er/nspe3/ao6nspe3.pdf

Maldonado González, A. L., González Gaudiano, E. J.y Cruz Sánchez, G. E. (2017). Una aproximación a la representación del cambio climático en habitantes de dos cuencas del estado de Veracruz, México. Revista Pueblos y Fronteras Digital, 12(23), 149-174. https://doi.org/10.22201/cimsur.18704115e.2017.23.291

Medina, I. F. y Páramo, P. (2014). La investigación en educación ambiental en América Latina: un análisis bibliométrico. Revista Colombiana de Educación, 66, 55-72. http://www.scielo.org.co/pdf/rcde/n66/n66ao3.pdf

Meira, P., Arto, M. y Montero, P. (2009). La sociedad ante el CC. Conocimientos, valoraciones y comportamientos en la población española. Fundación Mapfre. http://www.mapfre.com/ccm/content/documentos/fundacion/prev-ma/cursos/la-sociedad-ante-el-cambio-climatico.pdf abril 2019

Mochizuki, Y.y Bryan, A. (2015). Climate change education in the context of education for sustainable development: Rationale and principles. Journal of Education for Sustainable Development, 9(1), 4-26. https://doi. org/10.1177/0973408215569109

Monroe, M., Plate R., Oxarart A., Bowers A. y Chaves W. A. (2017). Identifying effective climate change education strategies: A systematic review of the research. Environmental Education Research, 25(6), 791-812. https:// doi.org/10.1080/13504622.2017.1360842

Moreno-Cano, A., Casado del Río, M. y Jiménez-Iglesia, E. (2015). Estudio sobre la percepción social del concepto de cambio climático y su divulgación en los medios de comunicación en la región de Santander-Colombia. Razón y Palabra, 91. https://www.revistarazonypalabra.org/index.php/ryp/article/view/95/ 
Olmos Martínez, E., Contreras-Loera R.y Gómez-Cabrera, D. (2016). El cambio climático: una perspectiva de género. Opción, 32(13), 1136-1157. https://dialnet.unirioja.es/servlet/articulo?codigo=5844707

Paniagua, E. C.y Hernández, D. (2013). La perspectiva del CC en el departamento de Antioquia. Trilogía, 9, 115-130. https://doi.org/10.22430/21457778.390

Pedrini, A., Brotto, D., Santos, V., Lima, L. y Nunes, R. (2016). Percepção ambiental sobre as mudanças climáticas globais numa praça pública na cidade do Rio de Janeiro (RJ, Brasil). Ciência \& Educação (Bauru), 22(4), 10271044. https://doi.org/10.1590/1516-731320160040012

Perkins, K. M., Munguia, N., Moure-Eraso, R., Delakowitz, B., Giannetti, B. F., Liu, G. y Velazquez, L. (2018). International perspectives on the pedagogy of climate change. Journal of Cleaner Production, 200, 1043-1052. https://doi.org/10.1016/j.jclepro.2018.07.296

Pinilla-Moscoso, C. I. y Puertas, D. C. (2017). Cambio climático, gestión y educación ambiental urbana: análisis geopolítico. Estudio de caso: Complejo de humedales en la localidad de 11-Suba, Bogotá. Redipe, 6(11), 65. https://revista.redipe.org/index.php/1/article/view/401

Ramírez-Vázquez, Y. y González-Gaudiano, E. (2016). Representaciones sociales del cambio climático en estudiantes de dos universidades veracruzanas. CPU-e. Revista de Investigación Educativa, 22, 1-27. http:// www.scielo.org.mx/scielo.php?script=sci_arttext\&pid=S1870-53082016000100002\&lng=es\&tlng=es

Reis, D. y Silva, L. (2016). Análise de dissertações e teses brasileiras de Educação Ambiental: compreensões elaboradas sobre o tema "mudanças climáticas". Ciência y Educação (Bauru), 22(1), 145-162. https://doi. org/10.1590/1516-731320160010010

Reis, D., Fernandes Silva, L. y Figueiredo, N. (2015). As complexidades inerentes ao tema "mudanças climáticas": desafios e perspectivas para o ensino de física. Ensaio Pesquisa em Educação em Ciências, 17(3), 535-554. https://www.scielo.br/pdf/epec/v17n3/1983-2117-epec-17-03-00535.pdf

Rosete-Vergés, F. (2014). Educación ambiental y cambio climático necesidad de desarrollar y fortalecer capacidades locales. Revista Interamericana de Educación de Adultos, 36(1), 104-119. https://www.redaly c.org/ pdf/4575/457545087006.pdf

Soares, D. y Murillo-Licea, D. (2013). Gestión de riesgo de desastres, género y cambio climático. Percepciones sociales en Yucatán, México. Cuadernos de Desarrollo Rural, 10(72), 181-199. http://www.scielo.org.co/scielo. php?script=sci_arttext\&pid=S0122-14502013000300010\&lng=pt\&tlng=es

Sterman, J. (2011). Communicating climate change risks in a skeptical world. Climatic Change,108(4), 811. https:// doi.org/10.1007/s10584-011-0189-3

Taber, F. y Taylor, N. (2009). Climate of concern-a search for effective strategies for teaching children about global warming. International Journal of Environmental and Science Education, 4(2), 97-116. https://www. pegem.net/dosyalar/dokuman/138326-20131230115857-1.pdf 
Unesco (2009). Report of the Unesco International Seminar on Climate Change Education, 27-29 de julio, París. [Portal web] https://www.unesco.org/science/doc/cc/CC_seminar_report_071209.pdf

Unesco (2010). The Unesco Climate Change Initiative: Climate Change Education for Sustainable Development. París. [Documento web] https://unesdoc.unesco.org/images/o019/001901/190101E.pdf

Unesco y unFCCC - United Nations Framework Convention on Climate Change (2016). Action for climate empowerment: Guidelines for accelerating solutions through education, training and public awareness. [Documento web] https:// unfccc.int/files/cooperation_and_support/education_and_outreach/application/pdf/action_for_climate_ empowerment_guidelines.pdf

Unesco (2017). Unesco at COP23. Climate change education. [Portal web] http://unesdoc.unesco.org/ images/0026/002600/260083e.pdf

Valerio-Hernández, V., Arguedas-Quirós, S. y Aguilar-Arguedas, A. (2016). Educación ambiental en el marco de una estrategia participativa para atender el cambio climático a nivel local: Experiencias en Costa Rica. Revista de Ciencias Ambientales, 49(2), 1-12. https://doi.org/10.15359/rca.49-2.1 
